\title{
A Method to Reduce Inter-ISP Transit Cost Caused by Overlay Routing Based on End-to-End Network Measurement
}

\author{
Kazuhito MATSUDA $^{\dagger \mathrm{a})}$, Go HASEGAWA ${ }^{\dagger \dagger \mathrm{b})}$, Satoshi KAMEI ${ }^{\dagger \dagger \mathrm{cc}}$, and Masayuki MURATA $^{\dagger \mathrm{d})}$,Members
}

\begin{abstract}
SUMMARY Overlay routing is an application-level routing mechanism on overlay networks. Previous researches have revealed that the overlay routing can improve user-perceived performance. However, it may also generate traffic unintended by ISPs, incurring additional monetary cost. In addition, since ISPs and end users have their own objectives respectively regarding traffic routing, overlay routing must be operated considering both standpoints. In the present paper, we propose a method to reduce interISP transit costs caused by overlay routing from the both standpoints of ISPs and end users. To determine the relationships among ASes, which are required for ISP cost-aware routing, we construct a method to estimate a transit cost of overlay-routed paths from end-to-end network performance values. Utilizing the metric, we propose a novel method that controls overlay routing from the both standpoints of ISPs and end users. Through extensive evaluations using measurement results from the actual network environments, we confirm that the advantage of the proposed method whereby we can reduce the transit cost in the overlay routing and can control the overlay routing according to the objectives of both ISPs and end users. key words: overlay network, overlay routing, inter-ISP transit cost, PlanetLab, multiple regression analysis
\end{abstract}

\section{Introduction}

Overlay routing is an application-level routing mechanism on overlay networks that provides application-level routes for network application traffic. An early and typical example is the Resilient Overlay Network (RON) [1], in which each overlay node measures the end-to-end latency and packet loss ratio of the network paths to other nodes. The path originating from the node is determined for the overlay network traffic, which can be either a direct path to the destination node or a relay path that traverses other node(s) before reaching the destination node as depicted in Fig. 1. In the present paper, the terms overlay routing and $I P$ routing are used to refer to traffic routing at the application-level and the IP-level, respectively.

One advantage of overlay routing is that user-perceived network performance, such as end-to-end latency and avail-

\section{Manuscript received April 20, 2012}

Manuscript revised September 21, 2012.

†The authors are with the Graduate School of Information Science and Technology, Osaka University, Suita-shi, 560-0871 Japan.

${ }^{\dagger}$ The author is with Cybermedia Center, Osaka University, Toyonaka-shi, 560-0043 Japan.

${ }^{\dagger \dagger}$ The author is with NTT Service Integration Laboratories, Musashino-shi, 180-8585 Japan.

a)E-mail: k-matuda@ist.osaka-u.ac.jp

b)E-mail: hasegawa@cmc.osaka-u.ac.jp

c) E-mail: kamei.satoshi@lab.ntt.co.jp

d)E-mail: murata@ist.osaka-u.ac.jp

DOI: 10.1587/transinf.E96.D.289 able bandwidth, can be improved without modifying the current IP network [2]-[4]. Such performance improvement is caused primarily by the policy mismatch between IP routing and overlay routing. IP routing is based primarily on metrics such as router-level and Autonomous System (AS)-level hop counts. In addition, Internet Service Providers (ISPs) that operate IP routing make their decisions based mainly on monetary contracts with their neighboring ISPs, which are either transit or peering relationships. Transit links and peering links have different monetary charging mechanisms for traffic exchange, and each ISP configures their routing according to differences. Conversely, overlay routing operated by end users primarily chooses the paths in a manner so as to enhance user-perceived performance. Consequently, user-perceived performance is improved by overlay routing.

Although this policy mismatch improves end-to-end network performance, it generates a problem for the ISPs' cost structure. Specifically, the inter-ISP transit cost (just as transit cost in the remainder of this paper) is increased over the entire network [5], [6]. To reduce transit cost, the locality-aware method has been proposed in [7] that controls network traffic based on the locality inferred from the IP address prefix or domain name. However, those types of information are not always suitable for estimating the locality of the Internet topology. Application-layer Traffic Optimization (ALTO) [8], which is based on the concept of P4P [9] is another approach that attempts to reduce transit cost by controlling outgoing traffic from an ISP while considering the utilization of its connected transit and peering links. However, such a mechanism can only optimize outgoing traffic from a single ISP, and it cannot control incoming traffic. Moreover, that mechanism cannot optimize the end-to-end network traffic governed by multiple interconnected ISPs. To reduce transit cost across the entire network, a routing mechanism based on transit cost information between ISPs on end-to-end paths is required. However, the contract information between ISPs is not available in general and a simple end-to-end measurement or estimation method to obtain this information has yet to be developed.

Even when such control mechanism of overlay routing is realized by ISPs or end users, the issue of policy mismatch is again raised. That is, when end users control the overlay network based on their own objectives, it may degrade the satisfaction of ISPs, and vice versa. Therefore, a novel method is required that considers the objectives of both ISPs and end users.

In the present study, we propose a novel method to de- 


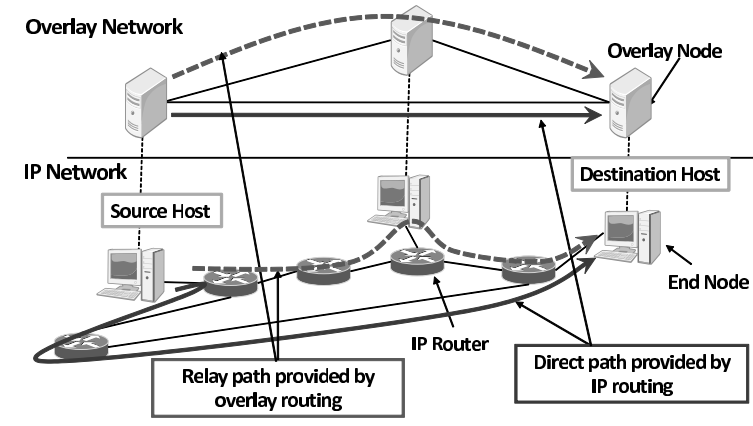

Fig. 1 Overlay routing.

crease the transit cost of overlay routing while accounting for the standpoints of end users and ISPs, which we call a limited overlay routing. The proposed method chooses overlay-routed paths using a transit cost metric of the paths. We propose two types of path selection methods for the limited overlay routing, which target end users and ISPs objectives, respectively.

The limited overlay routing needs the transit cost metric of overlay-routed paths. For this purpose, we build up a method to estimate the transit cost of overlay-routed paths from end-to-end network performance values that can be measured easily by overlay nodes, such as router-level hop count, end-to-end latency and available bandwidth. The estimation method is based on multiple regression analysis of network performance values.

We demonstrate the effectiveness of the proposed method by evaluating the performance of the overlay routing that is assumed to be operated on overlay networks on a PlanetLab [10] and a Japanese commercial network environments. To set a baseline for the discussion, we first evaluate the performance improvement of the overlay routing without a limitation on the transit cost metric. Next, we evaluate the limited overlay routing using precise information on the types of inter-AS links. Then, we show the regression equations used to estimate value of the transit cost metric for both environments. After that, we evaluate the performance of the limited overlay routing by using the proposed estimation method and discuss parameter settings from the standpoints of ISPs and end users.

The remainder of the present paper is organized as follows. In Sect. 2, research background on overlay routing is given and the problem of increased transit cost and incentives for reducing it are described. In Sect. 3, we propose a method to reduce transit cost. In Sect. 4, we explain the dataset used for evaluation of the proposed method, and then we present the results of the evaluation in Sect. 5. Finally, in Sect. 6, we summarize our conclusions.

\section{Background on Overlay Routing}

\subsection{Effectiveness of Overlay Routing}

Overlay routing can improve end-to-end network performance by choosing the paths based on application-level

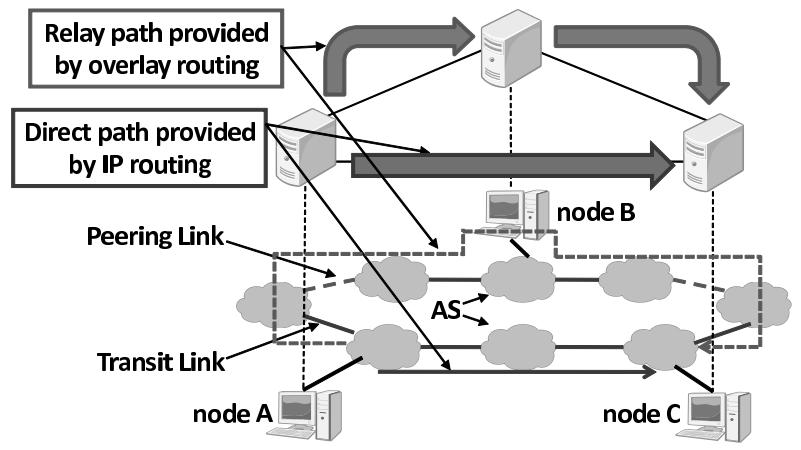

Fig. 2 Increase in number of traversed transit links by overlay routing.

network performance metrics, such as end-to-end latency, packet loss ratio, available bandwidth, and TCP throughput. This advantage of overlay routing is mainly a result of the policy mismatch between IP routing and overlay routing. Overlay routing typically makes their routing decisions that improve user-perceived performance using these metrics. Conversely, IP routing is based primarily on metrics such as router-level and AS-level hop counts, which do not always correlate to user-perceived performance.

In addition, ISPs have their own cost structures based on commercial contracts with their neighboring ISPs, and IP-level routing configurations are affected considerably by these cost structures. Two types of links are common between ASes $^{\dagger}$ : transit links that connect the upper-level and the lower-level ISPs, and peering links that are used for peering relationship. The monetary cost of the transit link is usually determined by the amount of traffic traversing the link, and transit links can be used by an ISP's customers. In contrast, there is almost no monetary charge for peering links, except for the cost paid to carrier companies for the physical link facilities. Therefore, peering links can be used only by traffic between interconnected ISPs.

Figure 1 shows a typical example of the advantage of overlay routing. We assume that IP routing uses the direct path and overlay routing chooses the relay path. The length of the arrows represents the value of the end-to-end latency, which is the sum of the propagation delays and delays caused by congestion at the routers. Comparing the direct and relay paths, the direct path has a lower router-level hop count but a higher end-to-end latency. Such a situation occurs, for instance, because of the congestion at the routers. Therefore, the overlay routing provides better userperceived performance (i.e., lower end-to-end latency) than the IP routing. For example, [11] showed from their evaluation results for a PlanetLab environment that overlay routing could reduce end-to-end latency in over $80 \%$ of end-to-end paths.

\subsection{Impact on the Cost Structure of ISPs}

Although overlay routing can improve user-perceived per-

${ }^{\dagger}$ We ignore sibling links because they connect ASes belonging to the same organization. 
formance, it may also generate traffic that does not follow to the ISPs' cost structure (i.e., the IP routing policy provided by the ISPs), and so the ISPs may incur additional monetary cost due to such traffic. If these cost increases accumulate, the transit cost over the entire network is increased.

Figure 2 shows a simple example of this problem in which three endhosts, all of which work as overlay nodes, are connected by overlay links each other. Each overlay link includes multiple inter-AS links, each of which is either a transit (solid-line) or peering (dashed-line) link. We assume that Node A generates traffic that is routed to Node $\mathrm{C}$. When using the IP or overlay routing that chooses the direct path, the traffic traverses two transit links. Conversely, when the overlay routing utilizes the relay path via Node B, the traffic traverses four transit links between Nodes A and B, and those between Nodes B and C. Therefore, the sum of the transit links traversed by the relay path is increased by two compared with the direct path and as a consequence, the transit cost over the entire network increases.

Naturally, there are possibilities that the relay path has lower transit cost than the direct path. However, we consider that the relay path usually has a higher transit cost because it is composed of a number of direct paths.

\subsection{Related Works}

The method proposed in [12] selects the overlay paths utilizing measurement results of capacity and available bandwidth. In [13], the authors present the method to construct and maintain overlay networks for improving user-perceived performance by selfish neighbor node selection. In [14], the authors propose QoS-aware overlay routing by balancing overlay traffic among overlay nodes. All of them target to improve user-perceived performance such as end-to-end latency and available bandwidth for end users' traffic, which is not specified for particular kinds of application. This feature is the same as that of our method proposed in this paper. However, the methods proposed in [12]-[14] are not treat the inter-ISP transit cost that incurs a considerable impact from the overlay routing as described in Sect. 2.2.

The method proposed in [15] uses a cost for overlay path creation and overlay traffic routing in an abstract way and optimizes the cost. In [16], the authors focus on the resource allocation on overlay networks and try to deal it as optimization problem. Although these methods can treat various kinds of cost by including it in their optimization problems, they have not considered the inter-ISP transit cost.

\section{Proposed Method}

We first explain the network model utilized in the present paper. Next, we propose a limited overlay routing with two path selection methods. One of those methods appropriate to the standpoint of end users and the other is for that of ISPs. Then, we present some use cases from both standpoints. Finally, we propose a method of estimating a transit cost metric from network performance values that can be

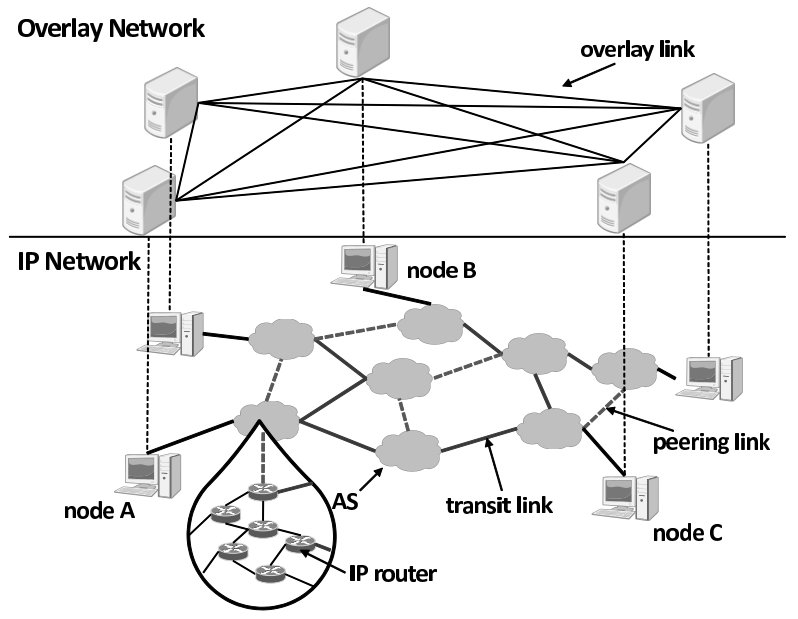

Fig. 3 Network model.

obtained easily.

\subsection{Network Model}

We assume the network model depicted in Fig. 3. The underlay network is constructed from a number of ASes, and each AS is constructed from a number of IP routers. Each $\mathrm{AS}$ is connected to its neighbors by transit or peering links. A transit cost is incurred whenever traffic traverses a transit link. Note that we ignore which ISPs connected by transit links are upper-level or lower-level ISPs, since we consider reduction in the transit cost over the entire network.

An overlay network is constructed over the underlay network and end hosts located at ASes perform as overlay nodes. We term overlay nodes just as nodes in the remainder of the present paper. We assume that the overlay routing can utilize the overlay links between all node pairs to evaluate the potential performance to reduce the transit cost due to the overlay routing. The construction method of the overlay network topology is beyond the scope of the present paper.

An overlay routing is operated on the overlay network and can provide a route from the source node to the destination node. We consider the following two types of overlayrouted paths.

direct path It is a path from the source node to the destination node that is routed directly. A direct path consists of only a single overlay link between the source and destination nodes, and so the direct path is equal to that provided by IP-level routing alone.

relay path It is a path from the source node to the destination node via another node. Here, we consider only two-hop paths, because paths with greater hop counts do not contribute to improve user-perceived performance [11]. Thus, a relay path consists of two overlay links. 


\subsection{Limited Overlay Routing}

The limited overlay routing can be implemented using any of the metrics associated with transit links. As a generalization, we describe the limited overlay routing using only a transit cost metric.

In what follows, $m_{i j}$ is the value of the transit cost metric for the overlay link between nodes $i$ and $j$. Hence, the value of transit cost metric for the direct path between nodes $i$ and $j$ and that of the relay path via node $k$ are given, respectively, as follows.

$$
\begin{aligned}
& M_{i j}=m_{i j} \\
& M_{i k j}=m_{i k}+m_{k j}
\end{aligned}
$$

\subsubsection{Improving User-Perceived Performance under Inter- ISP Transit Cost Constraint}

One path selection method focuses on the upper limit of increases in the value of transit cost metric. This selection method considers the end users' objectives. The constraint on the value of transit cost metric when choosing a relay path instead of a direct path is defined as follows.

$$
M_{i k j} \leq M_{i j}+\alpha
$$

where $\alpha$ is the upper limit of the increase in the value of transit cost metric through using the relay path. The overlay routing thus selects the relay path with the best performance from all possible candidates under this constraint. Here, the performance of direct path between nodes $i$ and $j$ is denoted $P_{i j}$, and the performance of relay path via node $k$ is denoted $P_{i k j}$. Then, we define the improvement ratio of user-perceived performance, which is denoted $\hat{I}_{i j}$, as follows.

$$
\begin{aligned}
& \hat{I}_{i j}=P_{i j} / \min _{k \neq i, j}\left(P_{i k j}\right) \\
& \hat{I}_{i j}=\max _{k \neq i, j}\left(P_{i k j}\right) / P_{i j}
\end{aligned}
$$

Here, Eq. (4a) is used in the case that a low performance metric value represents better performance, such as end-toend latency. Conversely, Eq. (4b) is used when a high value represents better performance, such as available bandwidth. Note that when no relay path has better performance than the direct path, the improvement ratio becomes smaller than one. In other words, the overlay routing with this path selection method provides the performance improvement for the data transmission between nodes under the limitation on the increase degree of the value of transit cost metric.

\subsubsection{Reducing Inter-ISP Transit Cost under User- Perceived Performance Constraint}

The other path selection method focuses on the decrease in the overlay routing performance. This method considers the
ISPs' objectives. When the best performance by the overlay routing between nodes $i$ and $j$ without considering the value of transit cost metric is provided by the relay path via node $l$, we define the constraints on the degree of decrease in overlay routing performance as follows.

$$
\begin{aligned}
& P_{i k j} \leq P_{i l j} \times(1+\beta) \\
& P_{i k j} \geq P_{i l j} \times(1-\beta)
\end{aligned}
$$

where $\beta$ determines the lower limit of the decrease degree of the performance of the overlay routing compared with the best performance. Note that when a low value represents better performance, Eq. (5a) should be satisfied, and when a high value represents better performance, Eq. (5b) should be satisfied. Then, the overlay routing selects a path with the lowest value of transit cost metric while satisfying Eqs. (5a) or (5b). The reduction in the value of transit cost metric, which is denoted as $\hat{M}_{i j}$, can be defined as follows.

$$
\hat{M}_{i j}=M_{i l j}-\min _{k \neq i, j}\left(M_{i k j}\right)
$$

In other words, this path selection method can reduce the value of transit cost metric under a given decrease in the user-perceived performance compared with that of the best path.

\subsection{Use Cases}

In this subsection, we present some use cases of proposed limited overlay routing.

\subsubsection{For End Users}

For the standpoint of end users, we assume the situation where the end users construct an overlay network by their end hosts as overlay nodes. Each overlay node exchanges the measurement results of network performance with other overlay nodes and each end user selects an overlay path independently. We can also presume another case where a content provider sets up overlay nodes on a number of ISPs and operates overlay networks to provide their contents to end users with high network performance.

In those cases, the end users can achieve the benefit, which is the improvement of network performance, provided by the overlay routing. For the case of content provider, they can increase their revenue from end users in return for better quality of content delivery. Hence, the end users and the content provider have incentive to operate it. On the other hand, the overlay routing focusing only to improve user-perceived performance may be harmful to ISPs because the overlay-routed traffic may generate additional transit cost. The considerable increase of transit cost causes ISPs to control or shut out the overlay-routed traffic. The proposed limited overlay routing in Sect.3.2.1 can resolve such situation by setting the upper limit of increase in transit cost generated by overlay-routed traffic. 


\subsubsection{For ISPs}

For the standpoint of ISPs, we suppose the case that a number of ISPs set up overlay nodes at own IP network. They share the measurement results of network performance among the ISPs and each ISP selects overlay paths for the end users belonging to the ISP. Alternatively, we can assume that the ISPs organize an alliance for overlay routing and the alliance operates the overlay routing in centralized manner. In both cases, after selecting the overlay paths, the ISPs or the alliance provide these paths to end users by an architecture such as ALTO.

In those cases, by using the cost-aware overlay routing, the ISPs mainly achieve the benefit, because that the ISPs can reduce the transit cost compared with the case that the end users select the overlay paths selfishly. However, if ISPs selects the overlay paths only focusing on the reduction of transit cost, the end users lost the incentive to use the overlay paths provided by the ISPs since such overlay paths do not improve user-perceived performance. The proposed limited overlay routing in Sect.3.2.2 can select the overlay paths maintaining the improvement of user-perceived performance while decreasing the transit cost.

\subsection{Transit Cost Estimation of an Overlay-Routed Path}

Although the limited overlay routing described above uses a transit cost metric of an overlay-routed path, such as the number of transit links, the exact value of the metric cannot be explicitly known by nodes because the contract information between ISPs is not disclosed in general. Furthermore, an effective method to measure the value of transit cost metric in an end-to-end manner has yet to be proposed. Indeed, in [17], the relationships among ISPs are inferred by collecting Border Gateway Protocol (BGP) messages from numerous backbone routers, which are also difficult to obtain by end users. In addition, the relationships between IP address prefix and AS numbers to obtain the AS-level paths are based on BGP messages. Although these information can be obtained at CAIDA [18] and Route Views Project [19] and we utilized them, it is unrealistic in the actual situations that the all overlay nodes obtain such information at such as CAIDA and Route Views Project. Furthermore, these information should be obtained periodically, because they change in time. Therefore, we propose a method of estimating the value of transit cost metric of an overlay link using network performance values that can be measured easily by nodes.

We first investigate the correlation between the true values of transit cost metric of paths between overlay nodes obtained by a method such as [17] and network performance values that are obtained easily by end-to-end measurement, such as router-level hop count, end-to-end latency, and available bandwidth. We find linear relationships between the number of transit links that have strong correlation with the true metric value of transit cost and each network performance value from the graph of the number of transit links vs. each network performance value using the PlanetLab dataset described in Sect. 4. Therefore, we utilize Pearson's correlation coefficient $C$ in Eq. (7).

$$
C=\frac{\sum\left(m_{i j}^{t}-\bar{m}^{t}\right)\left(x_{i j}-\bar{x}\right)}{\sqrt{\sum\left(m_{i j}^{t}-\bar{m}^{t}\right)^{2}} \sqrt{\sum\left(x_{i j}-\bar{x}\right)^{2}}}
$$

where $m_{i j}^{t}$ is the true value of transit cost metric of the overlay link between nodes $i$ and $j$, and $x_{i j}$ is each performance value (i.e., router-level hop count, end-to-end latency, and available bandwidth). $\bar{m}^{t}$ and $\bar{x}$ then represent the average values of each variable, respectively.

Then, to perform the estimation, we select some parameters that are highly correlated to the value of transit cost metric. We conduct a multiple regression analysis on the selected parameters and thus derive the regression equation from the analysis to estimate the value of transit cost metric.

We employ a linear least squares method to derive the regression equation. If $x_{i j}^{q}$ is the $q$-th parameter value of the overlay link between nodes $i$ and $j$, then the regression equation to estimate the value of transit cost metric of the overlay link, $m_{i j}^{e}$, is described as follows.

$$
m_{i j}^{e}=b_{0}+b_{1} x_{i j}^{1}+b_{2} x_{i j}^{2}+\ldots+b_{n} x_{i j}^{n}
$$

where $b_{0}$ is the intercept of the equation, $b_{q}$ is the partial coefficient value of the $q$-th parameter calculated by the multiple regression analysis, and $n$ is the number of parameters.

Once the regression equation is derived, the all overlay nodes can estimate the transit cost of overlay path by the network performance values that are easily obtained by themselves. In addition, the regression equation can be reused in other network environments if the property of the network environment is similar to where the regression equation is derived.

\section{Dataset}

To evaluate the proposed method, we utilize data obtained from two kind of actual network environments. One network environment is constructed from PlanetLab nodes, and the other from nodes located at Japanese commercial ISPs. To evaluate the overlay routing and the proposed method in both environments, we must know the following properties of the end-to-end path between overlay nodes: end-toend latency, available bandwidth, router-level path and hop count, and AS-level path and hop count. We also require the information on the transit/peering relationships between ASes to evaluate a value of transit cost metric of the overlay routing. In the remainder of this section, we describe both environments and explain how to obtain their property values.

\subsection{PlanetLab Environment}

For the PlanetLab environment, we obtained a dataset 
among the 459 PlanetLab nodes that were active when we obtained the measurement data. Actually, because a number of end-to-end paths were found for which we could not obtain the measurement data, we used the measurement data of 64077 end-to-end paths between nodes.

End-to-end latencies We obtained latencies of end-to-end paths between PlanetLab nodes from Scalable Sensing Service $\left(S^{3}\right)$ [20]. In $S^{3}$, the measurement results are available for all network paths between PlanetLab nodes, and are summarized every four hours. $S^{3}$ uses two types of end-to-end latencies, one is measured_latency that is actual measured values and the other is nv_estimated_latency that is estimated by the method proposed by Sharma et al. in [21]. Since measured_latency was not available for large part of node pairs, we utilized nv_estimated_latency in the present paper.

Available bandwidths They were obtained in the same way as end-to-end latencies. We utilized the results of available bandwidth measurements with Spruce [22] in $S^{3}$.

IP-level paths and router-level hop counts We conducted traceroute commands between all node pairs in PlanetLab. Here, we utilized the traceroute results obtained on November 12, 2008.

AS-level paths and AS-level hop counts We converted the IP-level paths into AS-level paths by using the relationships between IP address prefixes and AS numbers, which are available at the Route Views Project [19].

Transit/peering information To obtain a value of transit cost metric for each path, we used the information on transit/peering relationship between ASes that is available at CAIDA [18]. This information is obtained with the method in [17]. However, CAIDA does not provide the relationship information for all links between ASes. Furthermore, there are many IP addresses for routers whose corresponding AS numbers cannot be obtained by the method described above. Therefore, we applied two additional methods to infer the relationship information. The first methods is based on the degree of each AS (the number of outgoing links to other ASes). We first obtained the degree of each AS from CAIDA database and then derived the ratio at which the relationship was peering for each pair of degrees of ASes. Figure 4 depicts the distribution of the ratio for various pairs of ASes' degrees, where z-axis is the percentage of ASes pairs that have peering links in each cell. Then, the unknown relationship information was stochastically determined according the ratio distribution. The second method is based on the BGP property. When the AS number of the router on an IP-level path cannot be obtained by above-described method, this indicates that the BGP does not advertise the AS number of the router. This may mean that there is no need to advertise the number since the router belongs to the same AS at which the previous-hop router is located. For this

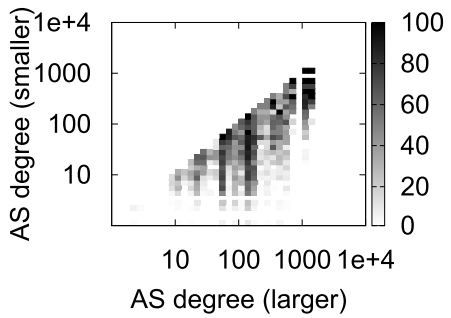

Fig. 4 Peering ratio from the degree of each AS pair.

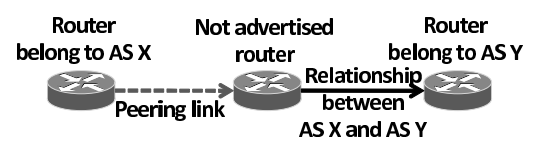

Fig. 5 Relationships inferred from BGP property.

Table 1 Average and variance values of network performance.

\begin{tabular}{|c||c|c||c|}
\hline \multirow{2}{*}{} & \multicolumn{2}{c||}{ PlanetLab } & Japanese commercial \\
\cline { 2 - 4 } & $\begin{array}{c}\text { end-to-end } \\
\text { latency }\end{array}$ & $\begin{array}{c}\text { available } \\
\text { bandwidth }\end{array}$ & $\begin{array}{c}\text { end-to-end } \\
\text { latency }\end{array}$ \\
\hline \hline average & $152 \mathrm{~ms}$ & $48,214 \mathrm{kbps}$ & $31 \mathrm{~ms}$ \\
\hline variance & $2.5 \times 10^{4}$ & $9.5 \times 10^{9}$ & $3.3 \times 10^{2}$ \\
\hline
\end{tabular}

Table 2 Average and variance values of AS-level degree.

\begin{tabular}{|c||c||c|}
\hline & PlanetLab & Japanese commercial \\
\hline \hline average & 27 & 21 \\
\hline variance & 12,061 & 1,028 \\
\hline
\end{tabular}

reason, as depicted in Fig. 5, when there exists an IPlevel path which is constructed of the router of AS X, the router whose AS number is not advertised, and the router of AS Y, the relationships between each router were estimated as peering and as the relationship between AS X and AS Y, respectively. Consequently, we could infer the unknown relationship between a nonadvertised router and AS Y's router once we had already obtained the relationship between AS X and AS Y.

To exhibit the characteristics of the environment, we show the average and variance values of end-to-end latencies and available bandwidth in Table 1. We also present the average and variance values of degrees of the ASes where the PlanetLab nodes are located in Table 2, which are calculated by the AS-level links observed in the traceroute results.

In the PlanetLab environment, we assumed two types of overlay networks. One network was the constructed from all nodes in the PlanetLab environment, which we call the full PlanetLab network. The other network, which we call the generalized PlanetLab network, was built such that the effect of geographical distribution of overlay nodes could be evaluated. The node distribution of the generalized PlanetLab network was constructed to conform to the Internet host distribution. To this end, we referred to the number of ASes in Regional Internet Registries (RIRs) in the current 
Table 3 Number of ASes in each RIR and number of nodes for evaluation.

\begin{tabular}{|c||c|c|}
\hline RIR (region name) & $\begin{array}{c}\text { number } \\
\text { of ASes }\end{array}$ & $\begin{array}{c}\text { number } \\
\text { of nodes }\end{array}$ \\
\hline ARIN (North America) & 24422 & 50 \\
\hline RIPE NCC (Europe) & 21065 & 43 \\
\hline APNIC (Asia) & 5782 & 12 \\
\hline LACNIC (South America) & 2815 & 6 \\
\hline
\end{tabular}

Internet obtained from [23], and to the number of PlanetLab nodes used in each region, which is proportional to the number of ASes (Table 3). We randomly selected PlanetLab nodes from each region according to Table 3. Comparing these overlay networks, we evaluate the effect of geographical node distribution on the proposed method in Sect. 5.4.

\subsection{Japanese Commercial Network Environment}

The dataset for the Japanese commercial network environment was obtained from a colleague. The environment is composed of 18 nodes of 13 Japanese commercial ISPs. The data of 289 end-to-end paths between nodes were used. This dataset included the full-mesh traceroute results and endto-end latencies measured using ping commands. Thus, end-to-end latencies, IP-level paths, and router-level hop counts could be determined from the dataset. The dataset utilized in the present paper was obtained on March 22, 2009. Note that we cannot obtain the data on available bandwidth, because that the measurement puts an extra load on the Japanese network environment. Then, the evaluation on the available bandwidth-based overlay routing is excluded from Sect. 5.

The average and variance values of end-to-end latencies and degrees of the ASes where the nodes are located are shown in Tables 1 and 2, respectively.

The AS-level paths and hop counts and transit/peering information were obtained in the same manner for the PlanetLab environment.

In the Japanese commercial network environment, we assume the overlay network constructed from all nodes in the Japanese commercial network environment, and we call this the Japanese network.

Because the dataset of Japanese commercial network environment is measured under non-disclosed conditions, we cannot describe the details of the geographical locations of the nodes in the environment. However, we ensure that the Japanese commercial network environment covers the wide area of Japan including large ISPs' network.

\section{Numerical Evaluation}

In this section, we first evaluate the performance improvement of overlay routing without a limitation on the transit cost metric in order to set a baseline for the discussion. Next, we evaluate the limited overlay routing using the precise information of relationships between ASes in order to confirm the potential performance improvement. After that, we present the regression equations, as explained in Sect.3.4, for the two networks on the PlanetLab and one networks on the Japanese commercial environment. Then, we show the evaluation results of the limited overlay routing by using the estimated transit cost value calculated through the regression equations. We also confirm the effect of the geographical node distribution on the proposed method.

We utilize end-to-end latencies and available bandwidths between nodes as path selection metrics for the overlay routing. We denote the end-to-end latency of the overlay link between nodes $i$ and $j$ as $\delta_{i j}$. Then the end-to-end latency of the direct path between the nodes denoted as $D_{i j}$ and that of the relay path via node $k$ denoted as $D_{i k j}$, are defined as follows.

$$
\begin{aligned}
& D_{i j}=\delta_{i j} \\
& D_{i k j}=\delta_{i k}+\delta_{k j}
\end{aligned}
$$

We do not explicitly include the processing cost of relaying traffic in Eq. (10) because this processing cost may be negligibly-small compared with propagation and congestion delays. To cite a case, the end-to-end latency of a relay path is approximately equal to the sum of the latencies of the direct paths that form the relay path in [24]. We denote the available bandwidth of the overlay link between nodes $i$ and $j$ as $\omega_{i j}$. Then the available bandwidth of the direct path between the nodes denoted as $B_{i j}$, and that of the relay path via node $k$ denoted as $B_{i k j}$, are defined as follows.

$$
\begin{aligned}
& B_{i j}=\omega_{i j} \\
& B_{i k j}=\min \left(\omega_{i k}, \omega_{k j}\right)
\end{aligned}
$$

We utilize Eqs. (4a) and (5a) as the improvement ratio and constraint on the performance of overlay routing, respectively, when end-to-end latency is employed as the routing metric, and Eqs. (4b) and (5b) when the available bandwidth is employed.

Since transit cost is generated by the traffic traversing the transit links, the cost is highly correlated to the number of transit links and the amount of traffic. We assume that the same billing mechanism is used for all transit links and that the traffic volumes between all overlay node pairs are equal. Based on these assumptions, when an overlay-routed path traverses transit links, the overlay-routed path costs one per transit link in the evaluation. We utilize the value calculated by this definition as the transit cost metric for the limited overlay routing. Of course, we can assume a specific billing mechanism for each transit link. However, because information is unavailable on how ISPs configure their billing mechanisms in practice, we use the simplest transit cost metric (i.e., one per transit link) in the evaluation.

We further assume that the value of transit cost metric based on the transit/peering information obtained by the method described in Sect. 4 is the true value of transit cost metric, because we consider that the transit/peering information reflects the actual network condition, since this information is acquired from numerous BGP routing tables and traceroute results. Moreover, the information has high 


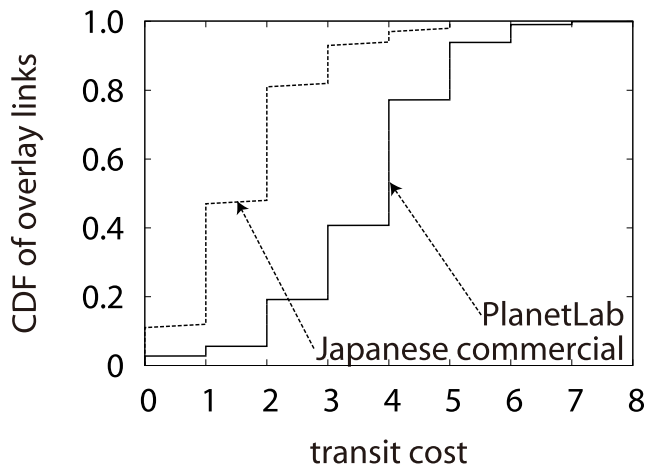

Fig. 6 Distribution of the true value of transit cost metric of overlay links in PlanetLab network environment and Japanese commercial network environment.

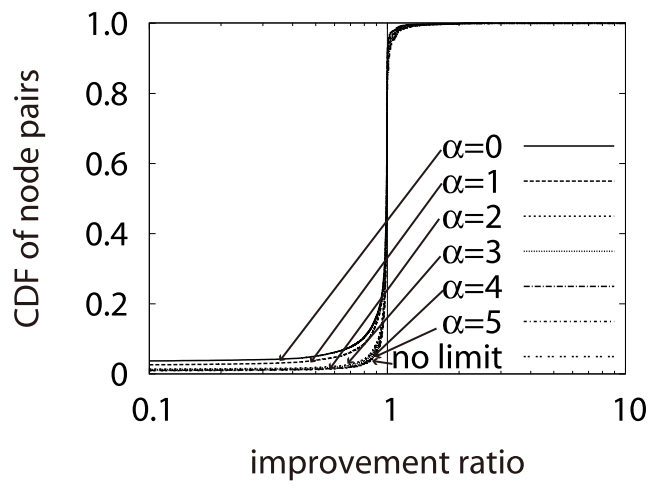

(a) end-to-end latency

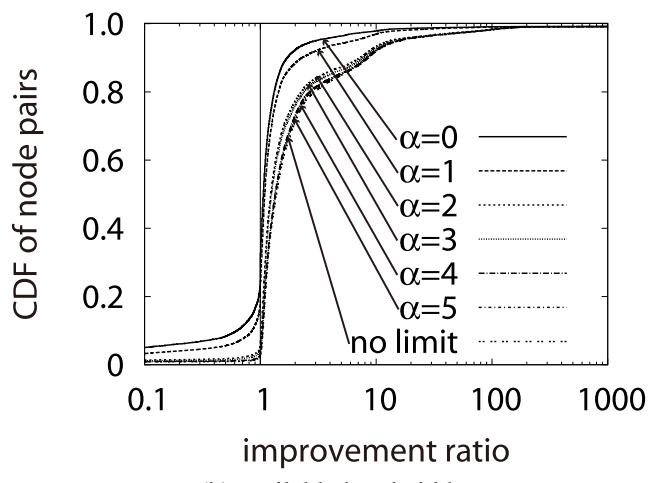

(b) available bandwidth

Fig. 7 Improvement ratio distribution with the limitation on the true value of transit cost metric (full PlanetLab network).

accuracy compared with the estimated value of transit cost metric calculated by Eq. (8), which is derived from only the network performance values easily obtainable by the nodes. The distribution of the true value of transit cost metric between PlanetLab nodes is shown in Fig. 6. We use this distribution as the baseline for the discussion in the evaluation.

\subsection{Unlimited Overlay Routing}

The "no limit" lines in Fig. 7 plot the cumulative distribution of the improvement ratios as defined in Eqs. (4) for the full PlanetLab network. Here, end-to-end latency is employed as the performance metric in Fig. 7 (a) and available bandwidth is employed in Fig. 7 (b). The results in Fig. 7 are based on the median value of dataset recorded over a two-week period from November 12, 2008 to November 25, 2008. The ratio of node pairs that have at least one relay path that has smaller end-to-end latency than the direct path is $22 \%$. In the case of available bandwidth, the percentage is $97 \%$. These results agree with the results in [11], implying that available bandwidth-based overlay routing improves user-perceived performance significantly.

The "no limit" line in Fig. 9 shows the result in the same manner as the line in Fig. 7 for the Japanese network. Since we do not have the data about available bandwidth for this environment, the evaluation is only on the end-to-end latency-based overlay routing. The ratio of node pairs that have at least one relay path that is better than the direct path is $15 \%$.

\subsection{Limited Overlay Routing with Precise Information on Transit Links}

Next, we show the results for the case when a limitation is placed on the true value of transit cost metric. This value is based on the precise relationship information among ASes obtained by the method explained in Sect. 4. The detailed algorithm of limited overlay routing can be found in Sect. 3.2.

Figure 7 exhibits the cumulative distribution of the improvement ratio for the full PlanetLab network when limiting the increase in the true value of transit cost metric. Path selection method here focuses on the upper limit of the increase in the value of transit cost, $\alpha$, by using Eq. (3). Note that when $\alpha$ is too small, relay paths satisfying the limitation cannot be found for some node pairs. These node pairs are accounted for at the origin of the $x$-axis. Figure 7 indicates that, no matter which routing metric is used, as $\alpha$ increases, the performance improvement of the overlay routing approaches that for the case without the limitation. Furthermore, when $\alpha$ is greater than or equal to three or four, the performance improvement of the overlay routing becomes approximately equal to the case without the limitation. From these results, we conclude that the overlay routing with the upper limit of the increase in the true value of transit cost metric can provide the performance improvement of the overlay routing similar to the case without the limitation, when the upper limit of the increase is greater than or equal to three or four. Figure 8 shows the cumulative distribution of the true value of transit cost metric for overlay-routed paths between all node pairs. These paths are the same as those chosen in Fig. 7 for the full PlanetLab network. This figure tells that when $\alpha$ is two in the overlay routing with the proposed method, the 80-th percentiles are 5.0 for the case of latency and 5.0 for the case of available bandwidth, respectively, whereas the values without the limitation are 6.0 and 6.4. When $\alpha$ is three, the 80-th percentiles are 5.5 for the case of latency and 7.0 for the case of available bandwidth. For the total transit cost of the all overlay-routed paths, when $\alpha=2$, the limited overlay rout- 


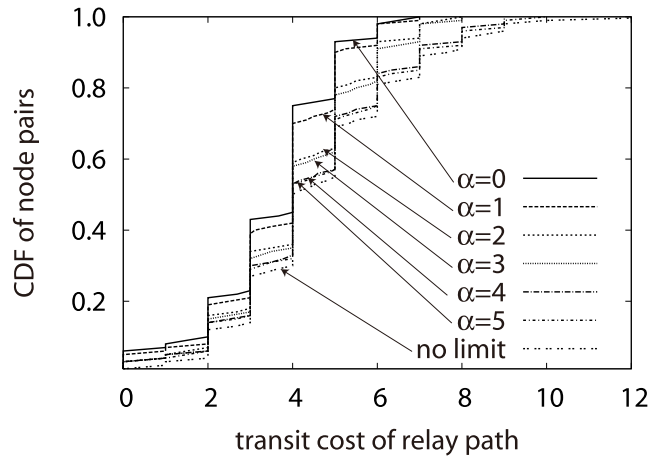

(a) end-to-end latency

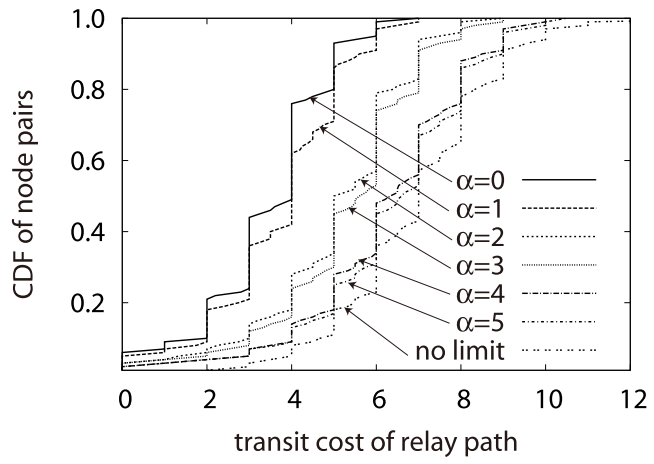

(b) available bandwidth

Fig. 8 Transit cost distribution with the limitation on the true value of transit cost metric (full PlanetLab network).

ing reduces the transit cost by $11 \%$ for the end-to-end latency and by $25 \%$ for the available bandwidth comparing the case without the limitation. When $\alpha=3$, these values are $9 \%$ for the end-to-end latency and $22 \%$ for the available bandwidth. Then, the limited overlay routing with the precise information can reduce transit cost to a certain degree, although this degree is small for the case of latency.

Figure 9 shows the results in the same manner as Fig. 7 for the Japanese network. The trend of the results is the same as that in the full PlanetLab network, which is that the performance improvement approaches that for the case without limitation as $\alpha$ increases, except one difference. That is, when $\alpha$ is greater than or equal to one (three or four for the full PlanetLab network), the performance improvement is approximately equal to that without the limitation. This lower value of $\alpha$ is due to the difference in the network property between both environments, that is, the true transit cost of paths in the Japanese commercial network environment is smaller than that in the PlanetLab environment. Figure 10 then shows the results in the same manner as Fig. 8 for the Japanese network. When $\alpha$ is one in the overlay routing with the proposed method, the 80-th percentile is 3.0 whereas the value without the limitation is 4.0 . For the total transit cost of the all overlay-routed paths, when $\alpha=1$, the limited overlay routing reduces the transit cost by $27 \%$. The same advantage as for the full PlanetLab network is thus revealed.

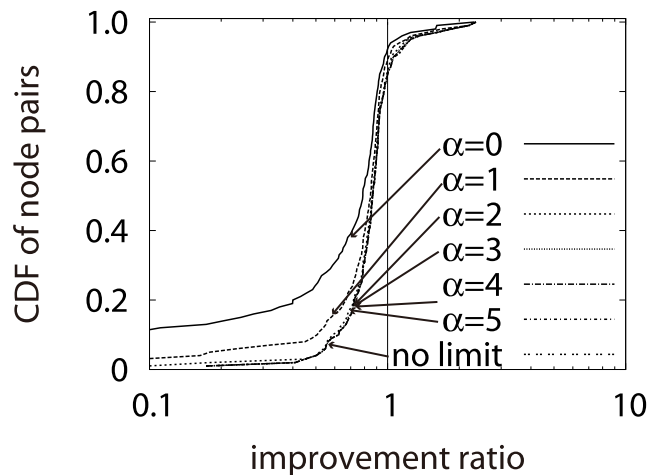

Fig. 9 Improvement ratio distribution with the limitation on the true value of transit cost metric (Japanese network).

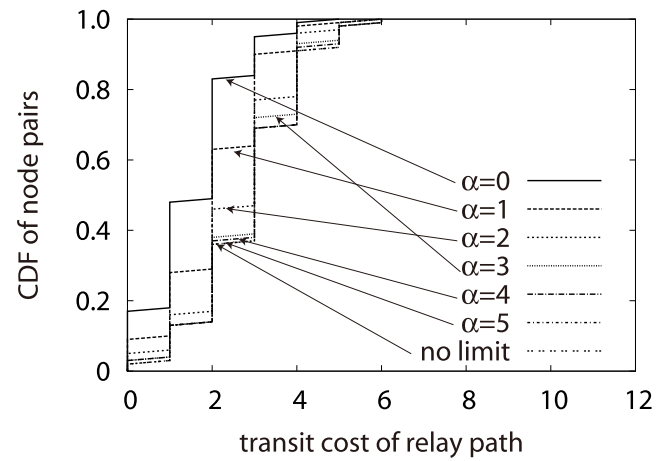

Fig. 10 True metric value of transit cost distribution with the limitation on the true value of transit cost metric (Japanese network).

Table 4 Correlation coefficients (full PlanetLab network).

\begin{tabular}{|c|c|}
\hline Router-level hop count & 0.420 \\
\hline End-to-end latency & 0.300 \\
\hline Available bandwidth & -0.027 \\
\hline
\end{tabular}

\subsection{Limited Overlay Routing with Estimated Value of Transit Cost Value}

\subsubsection{Regression Equations and Estimation Accuracy}

To evaluate the limited overlay routing with the proposed estimation method in Sect. 3.4, we first derived regression equations (Eq. (8)) for the three overlay networks described in Sect. 4. We calculated the correlation coefficients in Eq. (7) between the true value of transit cost metric and the following three metrics: router-level hop count, end-to-end latency, and available bandwidth for all nodes in the PlanetLab environment. The calculation results are listed in Table 4 , and based on these results, we omitted the available bandwidth from the regression equation because its correlation was quite weak compared with the other two metrics. Since we do not have available bandwidth data for the Japanese network, the same parameters (i.e., router-level hop count and end-to-end latency) were also selected in the regression equation. When the router-level hop count and the end-to-end latency of the overlay link between nodes $i$ 
Table 5 Partial coefficients of the regression equation.

\begin{tabular}{|c||c|c|c|}
\hline & $b_{y}$ & $b_{r}$ & $b_{d}$ \\
\hline Full PlanetLab network & 1.22 & 0.135 & 0.00263 \\
\hline Japanese network & -1.48 & 0.240 & -0.000889 \\
\hline Generalized PlanetLab network & 0.846 & 0.145 & 0.00120 \\
& $(0.20)$ & $\left(7.56 \times 10^{-4}\right)$ & $\left(1.08 \times 10^{-6}\right)$ \\
\hline
\end{tabular}

and $j$ are denoted as $h_{i j}$ and $\delta_{i j}$, respectively, then Eq. (8) can be rewritten as follows.

$$
\mu_{i j}^{e}=b_{y}+b_{r} h_{i j}+b_{d} \delta_{i j}
$$

where $b_{y}$ is the intercept of the equation, and $b_{r}$ and $b_{d}$ are the partial coefficients of the router-level hop count and the end-to-end latency, respectively. The partial coefficients $\left(b_{y}\right.$, $b_{r}$, and $b_{d}$ ), which are the results of the multiple regression analysis, for the three networks are listed in Table 5.

We also show the evaluation results of the estimation accuracy of the regression equation to verify the effectiveness of the analysis. The estimation error between the true and the estimated value of transit cost metric of an overlay link is calculated for each overlay node pair. The true value of transit cost metric of the overlay link between nodes $i$ and $j$ is denoted as $\mu_{i j}^{t}$, and the estimated value by the regression equation is demoted as $\mu_{i j}^{e}$. Then the estimation error of the overlay link, $d_{i j}$, is obtained as follows.

$$
d_{i j}=\mu_{i j}^{e}-\mu_{i j}^{t}
$$

Note that we consider both positive and negative values of the estimation errors. When the value is positive, it indicates that the transit cost is overestimated and the available relay paths are excessively restricted. Conversely, when the value is negative, it indicates that the transit cost is underestimated and the transit cost can be relaxed actually.

Figures 11 plot the cumulative distribution of $d_{i j}$ for all node pairs in each network environment. For comparison, the results of the single regression analyses on the routerlevel hop count and end-to-end latency are also plotted, respectively. The figures indicate that the maximum absolute estimation errors resulting from Eq. (8) are smaller than four for the PlanetLab environment, three for the Japanese commercial network environment. The absolute estimation errors are smaller than one for almost $60 \%$ of the overlay links in both network environments. Furthermore, compared with the results obtained by the single regression analyses, the multiple regression equation can give the highest estimation accuracy.

The differences between the regression equations for both environments can be observed in Table 5. These differences of network properties may be caused by the differences between the PlanetLab and the Japanese commercial network environments. PlanetLab is a global research network and is constructed from the nodes that are in universities and enterprises, whereas the Japanese commercial environment is constructed from the nodes located at Japanese commercial ISPs. In addition, PlanetLab nodes are spread more geographically than Japanese commercial environment. Hence, the proposed method can obtain the

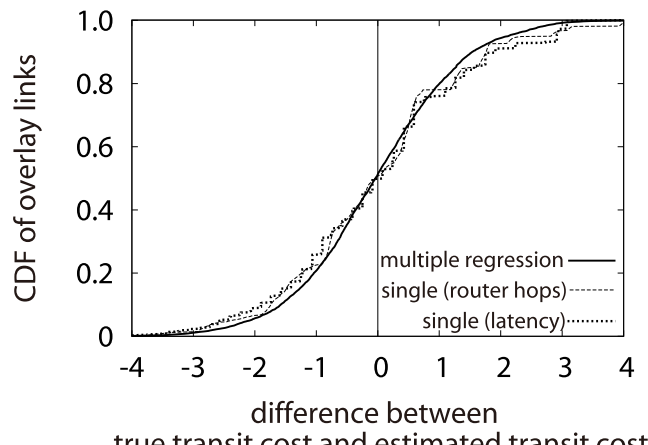

true transit cost and estimated transit cost (a) PlanetLab

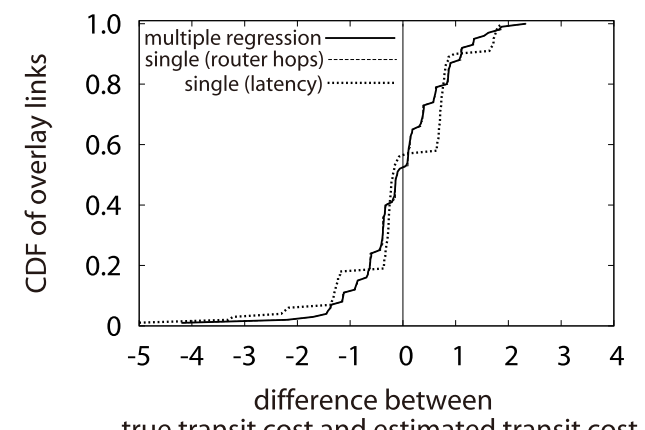

rue transit cost and estimated transit cost

(b) Japanese commercial network

Fig. 11 Estimation error distribution of the regression equation for all overlay links in each network environment.

regression equations appropriate to each network's properties.

\subsubsection{Improvement in User-Perceived Performance under Limitation on Inter-ISP Transit Cost}

Figure 12 plots the results in the same manner as Fig. 7 for the full PlanetLab network using the estimated value of transit cost metric instead of the true value. This figure tells that when $\alpha$ is smaller than three, many node pairs who do not have any relay path and the portion increases significantly compared with the results in Fig. 7, since a significant portion of the node pairs cannot find any relay paths satisfying the limitation. This is because of the estimation error described in Sect. 5.3.1. In contrast, when $\alpha$ is greater than or equal to three, the improvement is approximately the same as in the case without the limitation and that with the limitation on the true value of transit cost metric (Fig. 7). From these results, we conclude that the overlay routing with the proposed method, which has no precise information on transit links, can achieve the same performance as the case with the precise information. Figure 13 shows the results in the 


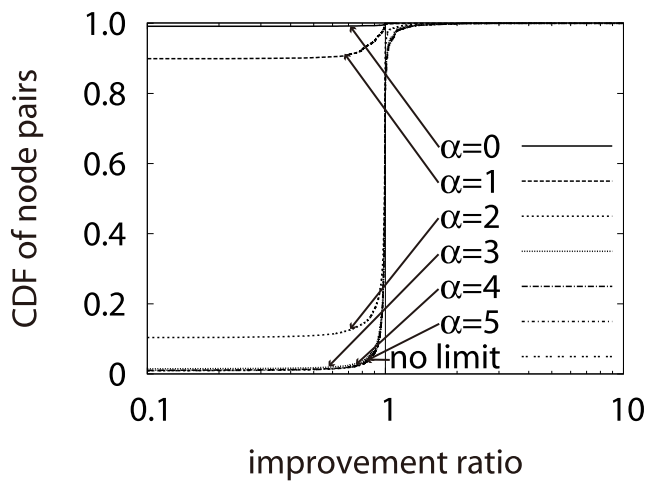

(a) end-to-end latency

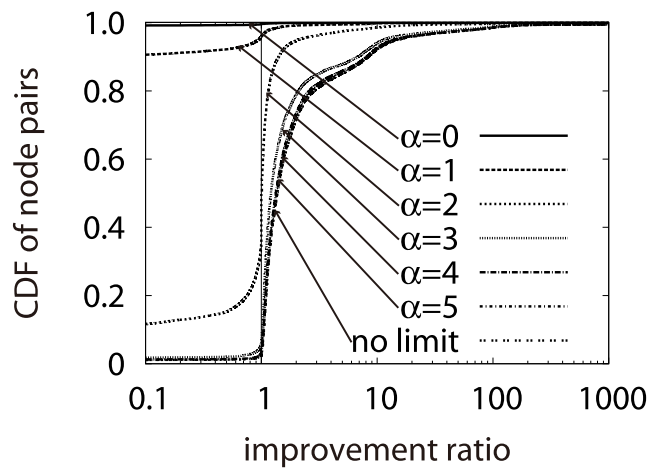

(b) available bandwidth

Fig. 12 Improvement ratio distribution with the limitation on the estimated value of transit cost metric (full PlanetLab network).

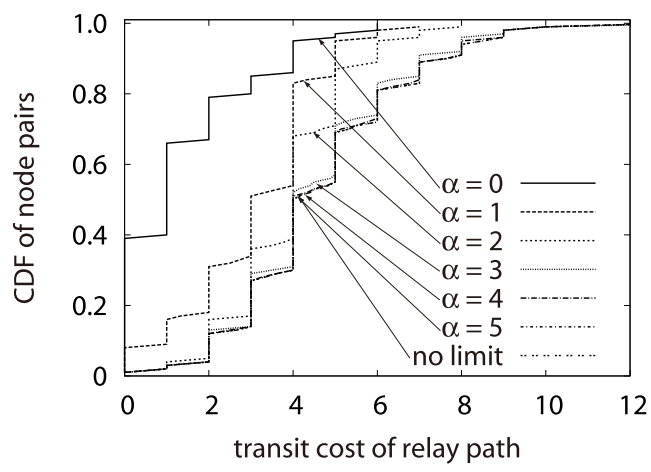

(a) end-to-end latency

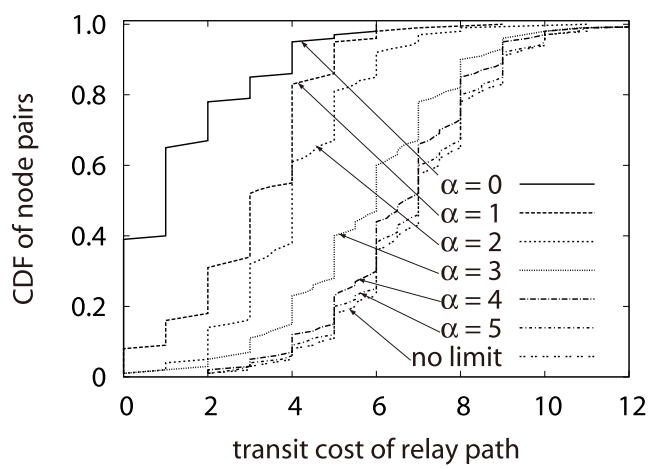

(b) available bandwidth

Fig. 13 Transit cost distribution with the limitation on the estimated value of transit cost metric (full PlanetLab network).

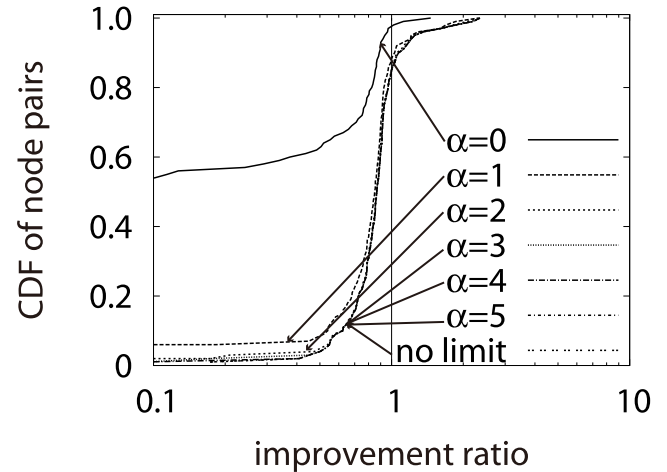

Fig. 14 Improvement ratio distribution with the limitation on the estimated value of transit cost metric (Japanese network).

same manner as Fig. 8 for the full PlanetLab network. This figure tells that when $\alpha$ is two in the overlay routing with the proposed method, the 80-th percentiles are 5.0 for the case of latency and 5.0 for the case of available bandwidth, respectively. When $\alpha$ is three, the 80 -th percentiles are 6.0 for the case of latency and 7.5 for the case of available bandwidth. For the total transit cost of the all overlay-routed paths, when $\alpha=2$, the limited overlay routing reduces the transit cost by $25 \%$ for the end-to-end latency and by $47 \%$ for the available bandwidth comparing the case without the limitation. When $\alpha=3$, these values are 3\% for the endto-end latency and $17 \%$ for the available bandwidth. Comparing Fig. 8 and 13, when $\alpha$ equals to zero or one, we can see that the limited overlay routing with the estimated transit cost excessively limits the transit cost, which is because of the estimation error of regression equation. However, we consider that these value of $\alpha$ is too tight limitation, which can be observed in Fig. 12. In the appropriate range of $\alpha$ (i.e., $\alpha$ is more than or equals to two) for the network performance, the limited overlay routing with the estimated transit cost reduce a certain transit cost while maintaining the network performance, though there are some estimation error of transit cost.

Figure 14 shows the results in the same manner as Fig. 12 for the Japanese network. The trend of the results is the same as that for the full PlanetLab environment except the exact value of $\alpha$. When $\alpha$ is greater than or equal to one, the overlay routing performance is approximately the same as the case with the true value of transit cost metric and the case without the limitation (Fig. 9). Fig. 15 then shows the results in the same manner as Fig. 13 for the Japanese network. When $\alpha$ is one in the overlay routing with the proposed method, the 80 -th percentile is 3.0 whereas the value without the limitation is 4.0. For the total transit cost of the all overlay-routed paths, when $\alpha=1$, the limited overlay routing reduces the transit cost by $18 \%$. The same advantage as for the full PlanetLab network is thus revealed.

From the viewpoint of the trade-off relationship between the performance improvement of overlay routing and the transit cost, Fig. 13 indicates that the greatest reduction in the true value of transit cost metric is when $\alpha$ is 


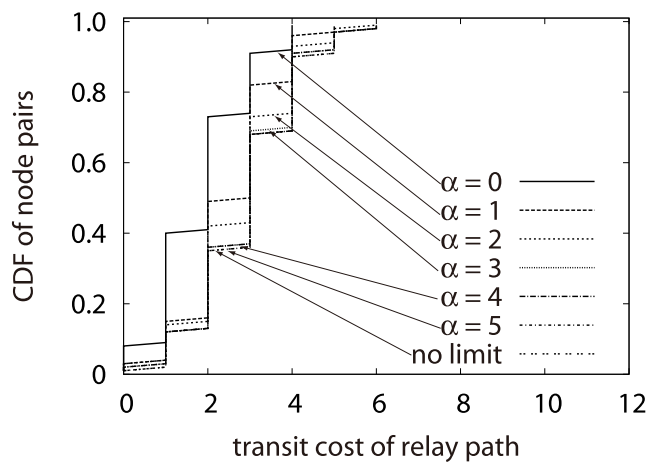

Fig. 15 True metric value of transit cost distribution with the limitation on the estimated value of transit cost metric (Japanese network).

equal to zero. However, the improvement ratio becomes less than one for a number of node pairs, implying that these node pairs cannot achieve any improvement in userperceived performance by the overlay routing. Conversely, when $\alpha$ is greater than or equal to three for the case of latency and four for the case of available bandwidth, almost no reduction is found in the true value of transit cost metric. We thus conclude that $\alpha=2$ for the case of latency is the best value from the viewpoint of the trade-off relationship between the performance of overlay routing and the reduction in the transit cost for the full PlanetLab network. For the case of available bandwidth, we conclude $\alpha=3$ is the best value.

Based on the results for both the PlanetLab network and Japanese commercial network environments, the parameter $\alpha$ may be affected by the scale of the target network environment. Specifically, for a large network, the more largest value we should choose for $\alpha$. The scale of network can be known through the IP-level or AS-level hop counts of end-to-end paths. In general, the parameter $\alpha$ can be set by the scale of target network and the degree of constraint required by the operator (i.e., end users).

\subsubsection{Reduction in Inter-ISP Transit Cost under Limitation on User-Perceived Performance}

Figure 16 shows the results of limited overlay routing with the path selection method that focuses on the degree of decrease in the performance of the overlay routing, where $\beta$ is the lower limit of the degree in Eqs. (5). Figures 16 (a) and 16 (b) plot the distribution of true value of transit cost metric reduction for the full PlanetLab network when the end-to-end latency and the available bandwidth employed as the routing metric, respectively. This figure indicates that the proposed method can reduce the true value of transit cost metric by at least one in $16 \%$ of node pairs when the endto-end latency is used as the routing metric and by at least one in $33 \%$ of node pairs when the case for available bandwidth, allowing only a $5 \%$ decrease in the overlay routing performance. We can achieve a greater reduction in the true value of transit cost metric by allowing a greater decrease in user-perceived performance. For example, if we can allow

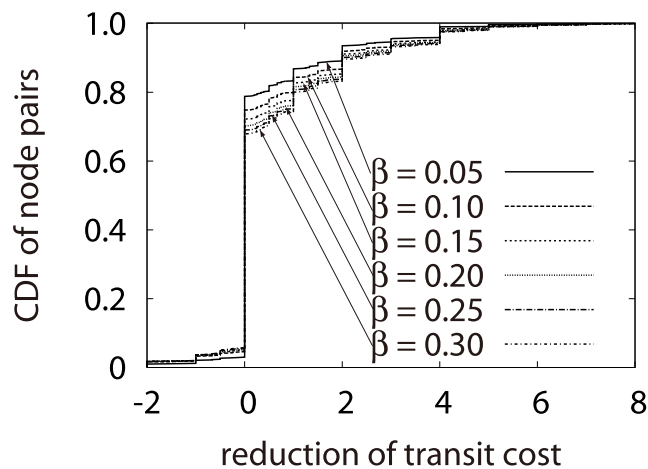

(a) End-to-end latency

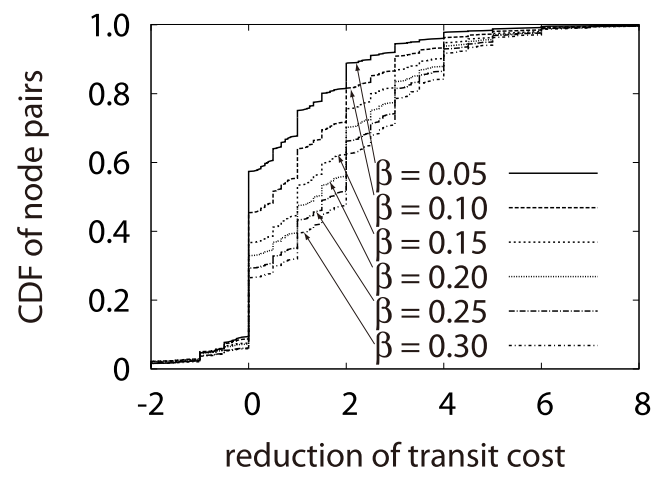

(b) Available bandwidth

Fig. 16 Distribution of reduction in the true value of transit cost metric with the limitation on the decrease in the overlay routing performance (full PlanetLab network).

a $30 \%$ decrease in the overlay routing performance, the true value of transit cost metric can be reduced by at least one in $25 \%$ and $68 \%$ node pairs when the end-to-end latency and the available bandwidth is employed as the routing metric, respectively.

Figure 17 shows the results in the same manner as Fig. 16 for the Japanese network. The trend of the results is the same as that for the full PlanetLab environment except the exact value of $\beta$. When allowing a 5\% decrease and a $30 \%$ decrease in the overlay routing performance, we can reduce the true value of transit cost metric by at least one in $17 \%$ and $42 \%$, respectively.

In practice, the parameter $\beta$ can be determined by the degree of constraint required by the operator (i.e., ISPs). For example, ISPs measure the performance of direct and relay paths and determine $\beta$ under the constraint such that the relay paths have sufficiently better than that of direct path.

\subsection{Effect of Geographical Distribution of Overlay Nodes}

We conducted node selections twenty times for the generalized PlanetLab network according to the method described in Sect. 4.1 and calculated the partial coefficients of the regression equation. The average and variance (in parentheses) values of the partial coefficients are listed in Table 5 . Since the variances are significantly smaller than the average values, we consider that the node selection in the gen- 


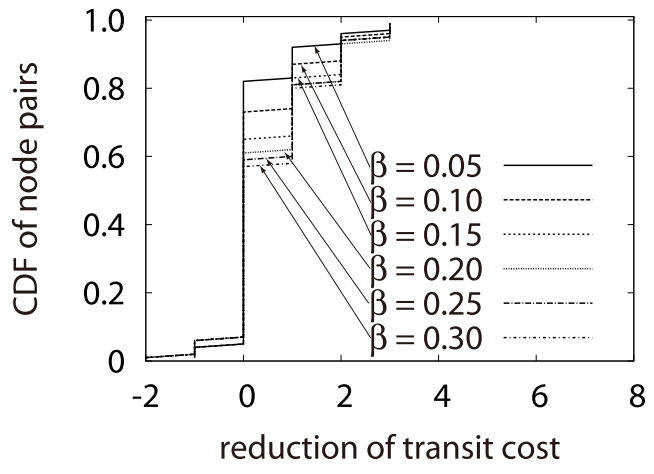

Fig. 17 Distribution of reduction in the true value of transit cost metric with the limitation on the decrease in the overlay routing performance (Japanese network).

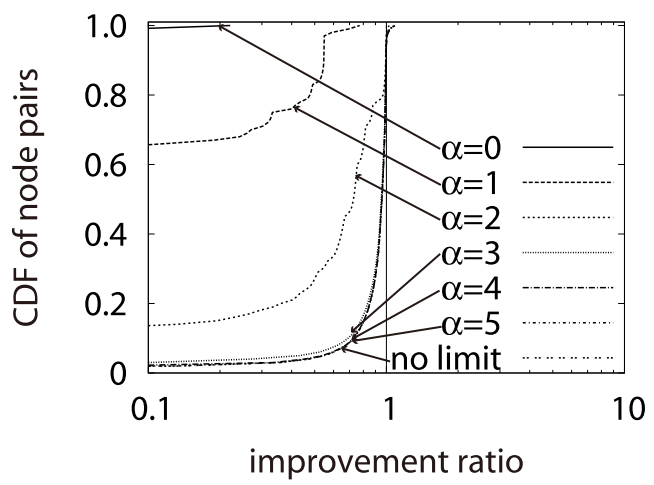

(a) End-to-end latency

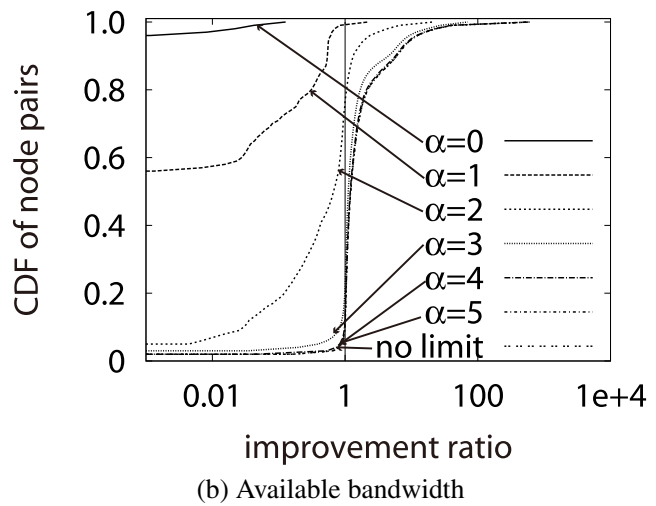

Fig. 18 Improvement ratio distribution with the limitation on the estimated value of transit cost metric (generalized PlanetLab network).

eralized PlanetLab network does not affect the performance of the proposed method.

Figure 18 plots the improvement ratio distribution of the overlay-routed paths for the generalized PlanetLab network. Comparing Fig. 12 and 18, the trend of the results is the same as that for the full PlanetLab network, especially when $\alpha$ is larger than or equal to three. From these results, we verify that the proposed method is effective for not only the overlay network constructed from all nodes in the PlanetLab environment, which are mainly located in North America and Europe, but also in a more general overlay network.
Since the trend of the results corresponding to Fig. 16 for the generalized PlanetLab environment is the same as that for the full PlanetLab environment, we do not show the results here.

\section{Conclusion}

In the present paper, we proposed a method to reduce transit costs caused by overlay routing while accounting for the objectives of both ISPs and end users. The proposed method utilizes a transit cost metric of an overlay-routed paths and chooses an overlay-routed path that can satisfy the objectives and constraints of both parties. Through the extensive evaluation using measurement results taken from the actual network environments, which were the PlanetLab and the Japanese commercial network environments, we confirmed the effectiveness of the proposed method. The results revealed that the advantage of the proposed method whereby we could estimate the transit cost of overlay-routed paths using measurable network performance values. Furthermore, the method could control the overlay routing according to the standpoints of end users and ISPs while reducing the transit cost over the entire network. Through the evaluation results, we confirmed the suitable parameter values for both network environments.

In general, the operator of the overlay routing with the proposed method can decide the degree of limitations based on their objectives and constraints. Using suitable parameters for a target network environment, the overlay routing with the proposed method can satisfy both of a reduction of transit cost and improvement of end-to-end network performance.

In the future, we intend to combine the proposed method with the architecture of ALTO in order to optimize the traffic on the entire network more efficiently.

\section{References}

[1] D.G. Andersen, H. Balakrishnan, M.F. Kaashoek, and R. Morris, "Resilient overlay networks," Proc. SOSP 2001, Oct. 2001.

[2] S. Banerjee, C. Kommareddy, K. Kar, B. Bhattacharjee, and S. Khuller, "Construction of an efficient overlay multicast infrastructure for real-time applications," Proc. INFOCOM 2003, April 2003.

[3] D.G. Andersen, A.C. Snoeren, and H. Balakrishnan, "Best-path vs. multi-path overlay routing," Proc. IMC 2003, Oct. 2003.

[4] C.L.T. Man, G. Hasegawa, and M. Murata, "Monitoring overlay path bandwidth using an inline measurement technique," IARIA International Journal on Advances in Systems and Measurements, vol.1, no.1, pp.50-60, Feb. 2008.

[5] P. Rodriguez, S.M. Tan, and C. Gkantsidis, "On the feasibility of commercial, legal P2P content distribution," SIGCOMM Computer Communication Review, vol.36, no.1, pp.75-78, Jan. 2006.

[6] S. Seetharaman and M. Ammar, "Characterizing and mitigating inter-domain policy violations in overlay routes," Proc. ICNP 2006, pp.259-268, Nov. 2006.

[7] T. Karagiannis, P. Rodriguez, and K. Papagiannaki, "Should Internet service providers fear peer-assisted content distribution?," Proc. IMC 2005, pp.6-6, Oct. 2005.

[8] IETF ALTO Working Group web site. available at http://datatracker.ietf.org/wg/alto/ 
[9] H. Xie, Y.R. Yang, A. Krishnamurthy, Y.G. Liu, and A. Silberschatz, "P4P: Provider portal for applications," SIGCOMM Computer Communication Review, vol.38, no.4, pp.351-362, Oct. 2008.

[10] PlanetLab web site. available at http://www.planet-lab.org/

[11] G. Hasegawa, Y. Hiraoka, and M. Murata, "Effectiveness of overlay routing based on delay and bandwidth information," IEICE Trans. Commun., vol.E92-B, no.4, pp.1222-1232, April 2009.

[12] S.J. Lee, S. Banerjee, P. Sharma, P. Yalagandula, and S. Basu, "Bandwidth-aware routing in overlay networks," Proc. INFOCOM 2008, pp.1732-1740, April 2008.

[13] G. Smaragdakis, V. Lekakis, N. Laoutaris, A. Bestavros, J.W. Byers, and M. Roussopoulos, "EGOIST: Overlay routing using selfish neighbor selection," Proc. CoNEXT 2008, pp.1-12, Dec. 2008.

[14] Z. Li and P. Mohapatra, "QRON: QoS-aware routing in overlay networks,” IEEE J. Sel. Areas Commun., vol.22, no.1, pp.29-40, Jan. 2004.

[15] M. Kamel, C. Scoglio, and T. Easton, "Optimal topology design for overlay networks," Proc. NETWORKING 2007, pp.714-725, May 2007.

[16] R. Cohen and D. Raz, "Cost effective resource allocation of overlay routing relay nodes," Proc. INFOCOM 2011, 2011.

[17] X. Dimitropoulos, D. Krioukov, M. Fomenkov, B. Huffaker, Y. Hyun, K. Claffy, and G. Riley, "AS relationships: Inference and validation," SIGCOMM Computer Communication Review, vol.37, no.1, pp.29-40, Jan. 2007.

[18] University of California CAIDA. available at http://www.caida.org/home/

[19] University of Oregon Route Views Project. available at http://www.routeviews.org/

[20] Hewlett-Packard Laboratories Scalable Sensing Service. available at http://networking.hpl.hp.com/s-cube/

[21] P. Sharma, Z. Xu, S. Banerjee, and S.J. Lee, "Estimating network proximity and latency," SIGCOMM Computer Communication Review, vol.36, no.3, pp.39-50, July 2006.

[22] J. Strauss, D. Katabi, and F. Kaashoek, "A measurement study of available bandwidth estimation tools," Proc. IMC 2003, pp.39-44, Oct. 2003.

[23] IANA, "IANA AS Numbers assignment data page." available at http://www.iana.org/assignments/as-numbers/

[24] R. Shohara, G. Hasegawa, and M. Murata, "Performance evaluation of spatial composition method of measurement results in overlay networks," IEICE Technical Report, IN2010-180, Feb. 2011.

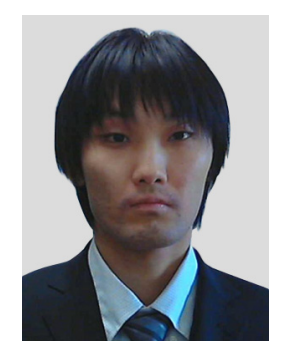

Kazuhito Matsuda received the M.E. degrees in Information Science and Technology from Osaka University, Japan, in 2010. He is now a doctoral student at the same school. His research work is in the area of overlay networks especially from the aspect of monetary cost.

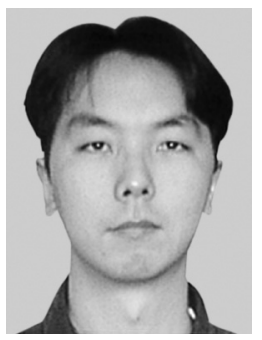

Go Hasegawa received the M.E. and D.E. degrees in Information and Computer Sciences from Osaka University, Japan, in 1997 and 2000, respectively. From July 1997 to June 2000, he was a Research Assistant of Graduate School of Economics, Osaka University. He is now an Associate Professor of Cybermedia Center, Osaka University. His research work is in the area of transport architecture for future highspeed networks and overlay networks. He is a member of IEEE and IPSJ.

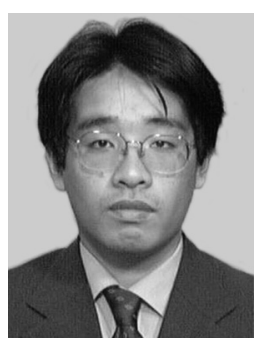

Satoshi Kamei was born in Hyogo, Japan in 1973. He received the B.E. and M.E. degrees from Kyoto University, Kyoto, Japan in 1997 and 1999, respectively. In 1999 he joined NTT Service Integration Laboratories, NTT corporation, Japan. He is engaged in research and development on Overlay networks.

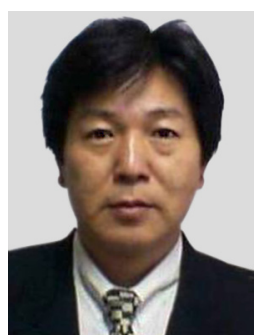

Masayuki Murata received the M.E. and D.E. degrees in Information and Computer Science from Osaka University, Japan, in 1984 and 1988, respectively. In April 1984, he joined Tokyo Research Laboratory, IBM Japan, as a Researcher. From September 1987 to January 1989, he was an Assistant Professor with Computation Center, Osaka University. In February 1989, he moved to the Department of Information and Computer Sciences, Faculty of Engineering Science, Osaka University. In April 1999, he became a Professor of Cybermedia Center, Osaka University, and is now with Graduate School of Information Science and Technology, Osaka University since April 2004. He has more than five hundred papers of international and domestic journals and conferences. His research interests include computer communication network architecture, performance modeling and evaluation. He is a member of IEEE and ACM. He is a chair of IEEE COMSOC Japan Chapter since 2009. Also, he is now partly working at NICT (National Institute of Information and Communications Technology) as Deputy of New-Generation Network R\&D Strategic Headquarters. 